\title{
Absorción de grasas termoxidadas. II. Influencia del nivel de alteración y porcentaje de grasa en la dieta
}

\author{
Por G. Márquez-Ruiz, M.C. Pérez-Camino, V. Ruiz-Gutiérrez y M.C. Dobarganes \\ Instituto de la Grasa y sus Derivados \\ Avda. Padre García Tejero, 4 - 41012 SEVILLA (España)
}

\section{RESUMEN}

Absorción de grasas termoxidadas. II Influencia del nivel de alteración y porcentaje de grasa en la dieta

En el presente trabajo se estudia la influencia de la alteración y porcentaje de grasa en la dieta sobre la digestibilidad de la grasa total as como de las fracciones insaponificable y de ácidos grasos no alterados.

La experiencia fue realizada utilizando 11 grupos de ratas Wista alimentadas con aceites de oliva no calentado y termoxidado. Se aplicaron técnicas analíticas estudiadas con anterioridad para la extracción y evaluación de los lipidos no absorbidos presentes en heces. nes:

Los resultados obtenidos permiten deducir las siguientes conclusio-

- La digestibilidad de la grasa disminuye con el incremento de alteración, pero es independiente del nivel de grasa en la dieta.

- La absorción aparente de ácidos grasos no alterados se encuentra afectada por la alteración de la grasa de la dieta.

- La alteración de la grasa ingerida contribuye al aumento de las cantidades excretadas de insaponificable de origen endógeno.

PALABRAS-CLAVE: Aceite termoxidado - Acido grado - Dieta - Digestibilidad - Insaponificable - Rata

\section{SUMMARY}

Absorption of thermoxidized fats. II. Influence of dietary alteration and fat level

In this paper, the influence of dietary fat alteration and level upon digestibilities of total fat, unsaponifiable matter and non-altered fatty acids, is studied.

11 groups of Wistar rats were fed diets containing non-heated and thermoxidized olive oils. Analytical techniques previously studied were used for isolation and evaluation of non-absorbed lipids present in feces.

The results obtained led to the following conclusions:

- Digestibility of fat decreases with dietary fat alteration but it is independent of the dietary fat level.

- The apparent absorption of non-altered fatty acids is affected by the dietary fat alteration.

- Dietary fat alteration significantly contributes to increase excreted levels of unsaponifiable from endogenous sources.

KEY-WORDS: Diet - Digestibility - Fatty acid - Rat - Thermoxidized oil - Unsaponifiable.

\section{INTRODUCCION}

El interés de los estudios nutricionales y toxicológicos de grasas termoxidadas está sobradamente justificado por la creciente utilización de la fritura como procedi- miento de preparación de alimentos y como nuevo proceso de fabricación de productos (Hauman, 1987; Orthoefer, 1987). Como consecuencia de la preocupación por las características higiénico-sanitarias de los aceites y grasas de fritura, en recientes normativas europeas se ha establecido un máximo del $25 \%$ de compuestos polares como límite de alteración para consumo humano (BOE, 1989; Firestone et al., 1991). Por otra parte, las grasas desechadas para consumo humano son frecuentemente utilizadas en nutrición animal (Steverink et al., 1985) y ello añade aún más interés al estudio de las propiedades fisiológicas y nutricionales de las grasas termoxidadas.

No obstante, una revisión de los trabajos realizados revela una gran divergencia en los resultados obtenidos (Márquez-Ruiz et al., 1990). En general, los estudios dirigidos a evaluar la absorción de grasas termoxidadas y los principales grupos de compuestos originados se han orientado según una doble vía:

a) La determinación directa de la absorción intestinal, mediante canulación linfática de los animales, que ha sido abordada en la mayoría de los casos utilizando productos de síntesis o aislados con un elevado grado de pureza (Iwaoka et al., 1978; Hsieh et al., 1977; Perkins et al., 1978; Combe et al., 1981).

b) La determinación cuantitativa de compuestos no absorbidos, que constituye una medida alternativa indirecta para la evaluación de las tasas de absorción.

En resumen, los resultados obtenidos en muy distintos estudios indican que la digestibilidad disminuye con el incremento de alteración de la grasa y esta observación está directamente relacionada con el incremento de los compuestos de polimerización (Potteau et al., 1970; Potteau et al., 1977)

Las divergencias encontradas entre los resultados de los distintos estudios se deben fundamentalmente a una insuficiente definición de las grasas utilizadas, exclusivamente basada en las condiciones de tratamiento. No hay que olvidar, sin embargo, que el tratamiento termoxidativo origina en las grasas una amplia serie de modificaciones que hace más difícil la determinación de los compuestos presentes. En este sentido, uno de los objetivos 
previos a este estudio fue establecer una metodología analítica que permita cuantificar los principales grupos de compuestos presentes tanto en las grasas ingeridas como en los lípidos no absorbidos (Márquez-Ruiz et al., 1990; Márquez-Ruiz et al., 1991).

En este trabajo se aborda el estudio de la influencia del nivel de alteración y porcentaje de grasa en la dieta sobre la digestibilidad de la grasa total así como de la fracción insaponificable y de los ácidos grasos no alterados. Para ello se han evaluado los lípidos fecales procedentes de ratas alimentadas con aceites de oliva no calentado y termoxidado, a diferentes niveles en la dieta.

\section{PARTE EXPERIMENTAL}

\subsection{Animales y dietas}

La experiencia se ha llevado a cabo con ratas macho de la cepa Wistar, con un peso inicial de $120 \mathrm{~g}$ aproximadamente, distribuidas en 10 lotes homogéneos constituidos por 4 animales cada uno.

Los animales fueron alimentados con una dieta base alipídica a la que se incorporó aceite puro de oliva, com- binando distintos valores para las dos variables de mayor interés: nivel de compuestos de alteración en la dieta y porcentaje de grasa en la misma. Los niveles de grasa seleccionados han sido 6,12 y $20 \%$ y los niveles de alteración los correspondientes al aceite de oliva puro no calentado, termoxidado a $180^{\circ} \mathrm{C}$ durante 150 horas y una mezcla de ambos al $50 \%$. La Tabla I recoge la composición de los aceites utilizados en los grupos de compuestos de interés, en este estudio: insaponificable, ácidos grasos no alterados y ácidos grasos polares. La composición de la dieta base se resume a continuación:

$\begin{array}{lr}\text { - Humedad } & 10,0 \% \\ \text { - Proteínas (caseína) } & 19,8 \% \\ \text { - Extracto libre de nitrógeno } & 57,6 \% \\ & \text { (glucosa + almidón) } \\ \text { - Celulosa } & 5,4 \% \\ \text { - Minerales } & 6,2 \% \\ \text { - Complejo vitamínico } & 1,0 \%\end{array}$

Se han controlado estrictamente las condiciones de preparación y conservación de las dietas. Con objeto de evitar su deterioro, se prepararon semanalmente y se envasaron en frascos herméticamente cerrados, con nitrógeno, que se conservaron en frigorífico $\left(4^{\circ} \mathrm{C}\right)$ hasta el momento de su administración a los animales.

Tabla I

Composición de los aceites de las dietas ( $\mathrm{g} / 100 \mathrm{~g}$ aceite)

\begin{tabular}{lccc}
\hline $\begin{array}{l}\text { Aceite } \\
\text { de oliva }\end{array}$ & Insaponificable & $\begin{array}{c}\text { Acidos grasos } \\
\text { no alterados }\end{array}$ & $\begin{array}{c}\text { Acidos grasos } \\
\text { polares }\end{array}$ \\
\hline No calentado & $0,89 \pm 0,06$ & $97,8 \pm 0,16$ & $1,2 \pm 0,21$ \\
No calentado: termoxidado $(1: 1)$ & $0,70 \pm 0,04$ & $79,2 \pm 0,39$ & $19,8 \pm 0,53$ \\
Termoxidado & $0,57 \pm 0,04$ & $63,4 \pm 0,29$ & $33,6 \pm 0,34$ \\
\hline
\end{tabular}

Valores medios \pm error standard de la media $(n=4)$.

Los animales se mantuvieron en jaulas de metabolismo individuales, que permiten cuantificar la ingesta y las excreciones fecal y urinaria, durante 15 días. A lo largo de este período, los animales ingirieron "ad libitum" agua y su dieta correspondiente, realizando un control riguroso de las cantidades ingeridas y excretadas diariamente.

El grupo de animales alimentado con dieta exenta de grasa estuvo sometido a control durante dos períodos de 15 días, con un intervalo de 2 meses entre ambos.

El seguimiento de los animales durante la experiencia ha incluido controles semanales de la evolución ponderal, signos externos y datos macroscópicos. Las heces fueron recogidas y pesadas diariamente, y mantenidas en congelador hasta el momento de su extracción.

\subsection{Análisis de lípidos no absorbidos}

Se aplica el esquema analítico propuesto en la primera parte de este trabajo (Márquez-Ruiz et al., 1991 b), para el aislamiento y separación de los lípidos no absorbidos, que incluye todas las modificaciones necesarias para garantizar una completa extracción de la grasa fecal y la inexistencia de alteraciones significativas.
En síntesis, los lípidos fecales obtenidos para cada animal en el período experimental de 15 días son extraídos con éter etílico (Toullec et al., 1968) en dos etapas, directamente y previa hidrólisis ácida. Tras la separación de la fracción insaponificable (Norma UNE 55-004-72) y acidificación de las soluciones alcohólicas de jabones, se obtienen los ésteres metílicos de ácidos grasos totales (Metcalfe et al., 1961) y se separan mediante cromatografía en columna de sílice (Dobarganes et al., 1984). La fracción no polar se eluye con hexano-éter etílico 95:5 y la elución de la fracción polar se realiza con éter etílico y cloroformo-metanol 2:1.

La separación e identificación de los ácidos presentes en la fracción no polar de lípidos endógenos se ha llevado a cabo mediante cromatografía gas líquido-espectrometría de masas (CGL-EM), en un aparato MS 30 B acoplado a un cromatógrafo Konik 019/375 utilizando una columna capilar Supelcowax $10(30 \mathrm{~m} \times 0,25 \mathrm{~mm}$ d.i.). La separación se hizo con temperatura programada desde $130^{\circ} \mathrm{C}$ a $200^{\circ} \mathrm{C}$, a $2^{\circ} \mathrm{C} / \mathrm{min}$ y 5 minutos de isoterma inicial. La temperatura del inyector fue $250^{\circ} \mathrm{C}$. El gas portador utilizado fue helio, con presión en cabeza de 10 psi. 


\subsection{Análisis estadístico}

Los resultados se expresan como valores medios \pm error standard de la media. Se ha aplicado un análisis de varianza sobre los coeficientes de digestibilidad corregida para detectar la existencia o no de diferencias significativas para las variables porcentaje y alteración de la grasa de la dieta y su interacción.

Las diferencias entre las medias de digestibilidad corregida para las diferentes dietas experimentales se analizaron mediante el test " $t$ " de Student $(P \leq 0,05)$.

\section{RESULTADOS Y DISCUSION}

\subsection{Análisis de lípidos endógenos}

La interferencia producida por los lípidos de origen endógeno en la evaluación cuantitativa de la grasa de la dieta no absorbida ha hecho necesaria la determinación de las cantidades globales excretadas y de los principales constituyentes de los lípidos fecales procedentes de animales alimentados con la dieta exenta de grasa. De esta forma, se pueden aplicar factores de corrección a los lípidos excretados por los diferentes grupos de animales.

La Tabla II recoge los resultados obtenidos para los lípidos endógenos totales, fracción insaponificable y ácidos grasos correspondientes a dos períodos experimentales de 15 días, al comienzo y al final de la experiencia. Puesto que no existen diferencias significativas, debido a la edad de los animales, se ha calculado la media de todos los valores así como sus límites de confianza. La excelente reproducibilidad encontrada es una garantía de que la corrección utilizando valores medios, no contribuye significativamente a incrementar el error, cuando se calculan las tasas corregidas de digestibilidad.

Como puede observarse, los lípidos de origen endógeno están fundamentalmente constituidos por componentes del insaponificable (62\%), que proceden en gran

Tabla II

Lípidos de origen endógeno: Cantidades excretadas durante 2 períodos experimentales (g/15 días).

\begin{tabular}{|c|c|c|c|c|c|}
\hline & Ratas & Total & $\begin{array}{c}\text { Fracción } \\
\text { insaponificable }\end{array}$ & $\begin{array}{c}\text { Acidos grasos } \\
\text { no alterados }\end{array}$ & $\begin{array}{l}\text { Acidos }^{*} \\
\text { polares }\end{array}$ \\
\hline & 1 & 0,42 & 0,26 & 0,16 & - \\
\hline 1er Período & 2 & 0,48 & 0,31 & 0,12 & 0,05 \\
\hline \multirow[t]{3}{*}{ Experimental } & 3 & 0,36 & 0,21 & 0,08 & 0,07 \\
\hline & 4 & 0,35 & 0,21 & 0,10 & 0,04 \\
\hline & 1 & 0,44 & 0,29 & 0,10 & 0,05 \\
\hline 2.ำ Período & 2 & 0,36 & 0,19 & 0,09 & 0,08 \\
\hline \multirow[t]{2}{*}{ Experimental } & 3 & 0,38 & 0,23 & 0,12 & 0,03 \\
\hline & 4 & 0,40 & 0,27 & 0,08 & 0,05 \\
\hline $\bar{x}$ & & 0,39 & 0,25 & 0,11 & 0,04 \\
\hline${ }^{\star \star *} S_{\bar{x}}$ & & 0,038 & 0,035 & 0,021 & - \\
\hline
\end{tabular}

- Calculados por diferencia a 100.

$* t=2,365$.

parte de las secreciones hepática e intestinal (Long et al., 1978; Miettinen et al., 1981), y por ácidos grasos (Hoet et al., 1963), mayoritariamente de baja polaridad (27\%).

La Figura 1 muestra un cromatograma representativo de los ésteres metílicos de ácidos no polares de origen endógeno, separados e identificados por CGL-EM. Este grupo de compuestos incluye un elevado número de ácidos de longitud de cadena comprendida entre 14 y 28 átomos de carbono, con predominio de ácidos saturados sobre insaturados, de cadena par sobre impar y de serie normal sobre los ramificados.

\subsection{Coeficientes de digestibilidad}

A partir del análisis de los lípidos no absorbidos se han calculado los valores de digestibilidad de la grasa total ingerida, así como de la,fracción insaponificable y los ácidos grasos no alterados.

El coeficiente de digestibilidad corregida o verdadera (DV) se calculó según la fórmula de Deuel (Deuel, 1957):

$$
\operatorname{DV}(\%)=\frac{\text { Ingerido }-(\text { Excretado }- \text { Endógeno })}{\text { Ingerido }} \times 100
$$

La Tabla III muestra los valores medios de lípidos ingeridos y excretados para todos los grupos de animales durante un período experimental de 15 días. En la tabla se recogen todos los datos correspondientes a los lípidos extraídos directamente (grasa neutra) así como las cantidades totales excretadas, incluyendo los lípidos obtenidos tras hidrólisis ácida y, finalmente, los valores 


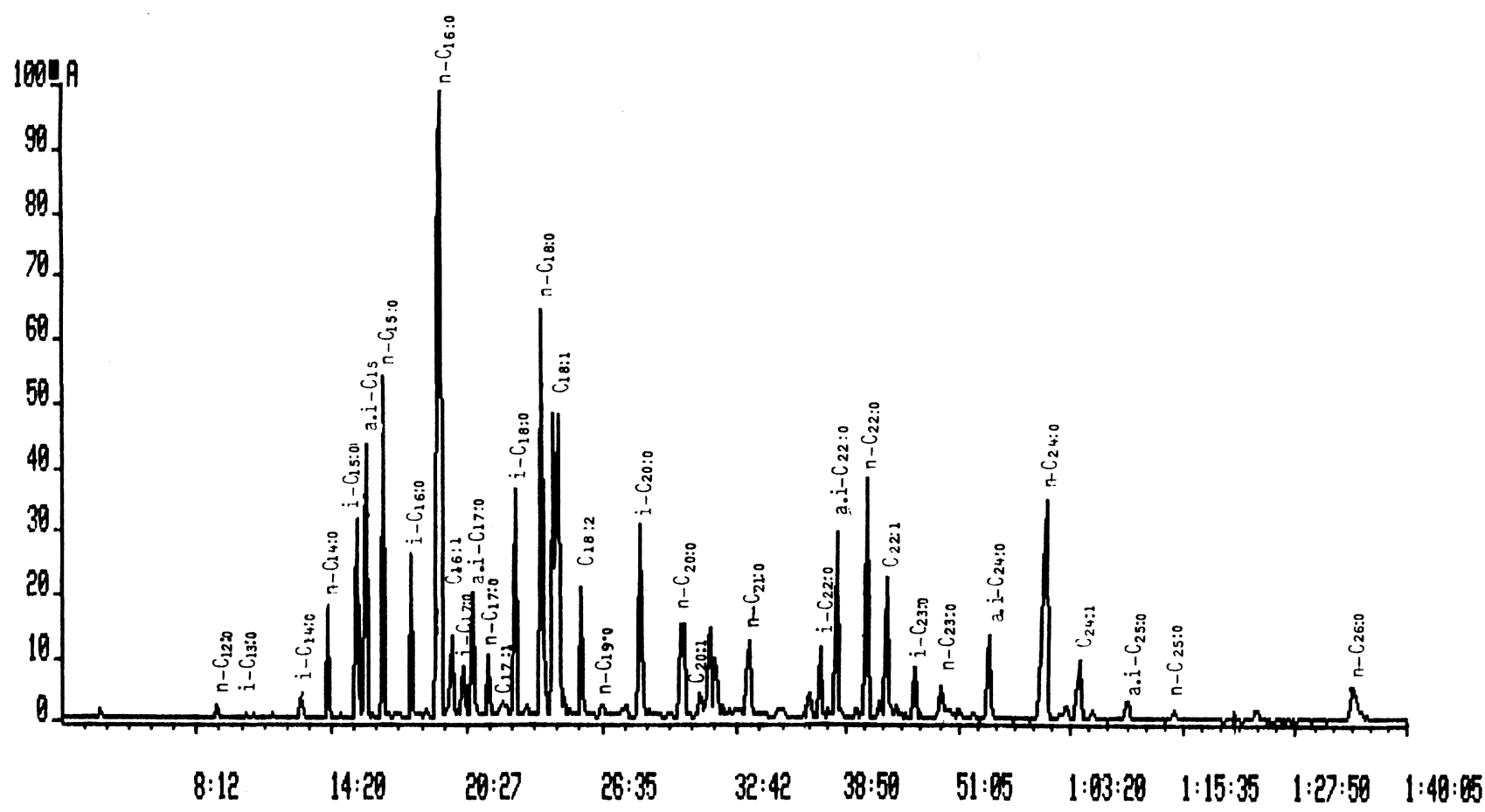

Figura 1

Esteres metilicos de ácidos no polares presentes en los lipidos de origen endógeno. Cromatograma de la corriente iónica total Abreviaturas: $n$-serie normal $i$ - serie iso a.i - serie anteiso

corregidos tras aplicar el factor correspondiente a la media de lípidos endógenos excretados en 15 días. La última columna incluye los coeficientes de digestibilidad corregida.

El análisis de varianza aplicado a los coeficientes de digestibilidad corregida indicó que la diferencia entre niveles de grasa no es significativa, pero la variación entre niveles de alteración lo es para $P<0,01$. Por otra parte, no existe interacción entre nivel de alteración y de grasa en la dieta.

Como era de esperar, las digestibilidades de las grasas no calentadas son muy elevadas mientras que las grasas más alteradas presentan unos valores particularmente bajos, que suponen un incremento de 7 a 10 veces en los lípidos no absorbidos con respecto a los grupos que ingirieron grasas no calentadas. Por otra parte, los resultados para la mezcla al $50 \%$ de grasa no calentada y alterada son intermedios y, aproximadamente, los correspondientes a la mezcla teórica. En consecuencia, los datos reflejan que la disminución de la digestibilidad está directamente relacionada con el incremento de la concentración en productos alterados en la dieta, hecho fácilmente comprensible si consideramos la participación de moléculas alteradas de alto peso molecular en las grasas termoxidadas, que podrian encontrar dificultades para atravesar la barrera intestinal.

En relación con la fracción insaponificable, la Tabla IV recoge las cantidades medias excretadas durante el período experimental así como los valores de digestibilidades corregidas.

En primer lugar, destaca especialmente que los coeficientes de digestibilidad sean negativos, a excepción de los correspondientes a aceites frescos. Es decir, las cantidades excretadas superan a las ingeridas, aún aplicando las correcciones en base a la participación endógena, lo que indica que dichas correcciones son inferiores al insaponificable endógeno realmente presente. Teniendo en cuenta este hecho y la proporción minoritaria de insaponificable en los aceites de las dietas, inferior al 1\%, no es extraño que se obtengan diferencias significativas dificiles de interpretar para los valores de digestibilidad.

Por otra parte, es interesante observar el incremento de las cantidades excretadas a medida que aumenta la alteración, aún cuando es en este sentido en el que disminuye el insaponificable ingerido en la dieta. La conclusión más evidente es que existe una estrecha relación entre los lípidos endógenos y la composición de la dieta. En estudios posteriores hemos analizado más profundamente este aspecto, a través de la cuantificación de dos grupos de compuestos de interés, ausentes en la dieta y, por tanto, exclusivamente relacionados con los lípidos de origen interno (Márquez-Ruiz, 1989).

La Tabla $V$ muestra las cantidades excretadas de ácidos grasos no alterados y los valores de digestibilidades corregidas.

En primer lugar, los coeficientes de digestibilidad son 
Tabla III

Lípidos totales: Cantidades ingeridas y excretadas durante 15 días. Digestibilidades corregidas

\begin{tabular}{|c|c|c|c|c|c|c|}
\hline \multicolumn{2}{|c|}{ Aceite en dieta } & \multirow{2}{*}{$\begin{array}{l}\text { Lípidos } \\
\text { ingeridos (g) }\end{array}$} & \multicolumn{3}{|c|}{ Lipidos excretados (g) } & \multirow{2}{*}{$\begin{array}{l}\text { Digestibilidad } \\
\text { corregida (\%) }\end{array}$} \\
\hline Alteración & Nivel (\%) & & Neutros & Totales & Corregidos & \\
\hline \multirow{3}{*}{ No calentado } & 6 & $18,78 \pm 0,70$ & $1,01 \pm 0,51$ & $1,31 \pm 0,22$ & $0,99 \pm 0,22$ & $94,8 \pm 0,5 a$ \\
\hline & 12 & $32,49 \pm 0,46$ & $0,87 \pm 0,05$ & $1,66 \pm 0,06$ & $1,35 \pm 0,06$ & $95,8 \pm 0,2^{a}$ \\
\hline & 20 & $35,26 \pm 1,08$ & $0,96 \pm 0,32$ & $1,74 \pm 0,38$ & $1,42 \pm 0,38$ & $96,5 \pm 0,5^{a}$ \\
\hline \multirow{3}{*}{$\begin{array}{l}\text { No calentado: } \\
\text { Termoxidado } 1: 1\end{array}$} & 6 & $18,17 \pm 0,50$ & $1,73 \pm 0,12$ & $2,92 \pm 0,08$ & $2,61 \pm 0,08$ & $85,6 \pm 0,6 b$ \\
\hline & 12 & $35,79 \pm 2,21$ & $3,70 \pm 0,18$ & $5,36 \pm 0,21$ & $5,04 \pm 0,21$ & $85,8 \pm 0,6 b$ \\
\hline & 20 & $45,56 \pm 2,89$ & $6,72 \pm 0,55$ & $7,74 \pm 0,58$ & $7,42 \pm 0,58$ & $83,7 \pm 0,4 b$ \\
\hline \multirow{3}{*}{ Termoxidado } & 6 & $17,88 \pm 0,70$ & $3,28 \pm 0,64$ & $5,42 \pm 0,36$ & $5,09 \pm 0,36$ & $71,6 \pm 1,2 c$ \\
\hline & 12 & $35,22 \pm 0,98$ & $8,79 \pm 0,43$ & $10,66 \pm 0,52$ & $10,34 \pm 0,52$ & $70,7 \pm 0,7 c$ \\
\hline & 20 & $48,12 \pm 2,57$ & $12,72 \pm 1,39$ & $14,73 \pm 1,41$ & $14,41 \pm 1,41$ & $70,2 \pm 1,8 \mathrm{c}$ \\
\hline
\end{tabular}

Valores medios \pm error standard de la media.

Los valores de digestibilidad con diferente superindice son significativamente diferentes $(P \leq 0,05)$.

muy elevados en todos los casos y siempre superiores a los obtenidos para los lípidos totales (Tabla II). Los valores más elevados corresponden a los grupos que consumieron aceite no calentado, con coeficientes muy próximos a 100. Sin embargo, no existen diferencias significativas debidas al porcentaje de grasa en la dieta. Finalmente, resulta particularmente interesante la disminución de la digestibilidad a medida que aumenta la degradación de la grasa ingerida ya que, teniendo en cuenta las elevadas tasas de absorción de los ácidos grasos, podría esperarse que su digestibilidad fuera independiente del nivel de alteración. Es importante comentar que la disminución de la digestibilidad de la grasa, como conse- cuencia del proceso termoxidativo, se ha atribuido a la dificultad de absorción de compuestos de polimerización (Potteau et al., 1970; Potteau et al., 1977). Sin embargo, la cuantificación realizada en este estudio permite deducir que a tal disminución contribuyen también los ácidos grasos no alterados.

Puesto que antes de la absorción propiamente dicha tiene lugar la hidrólisis del triglicérido y experimentos realizados "in vitro" muestran una considerable reducción en la acción de la lipasa pancreática sobre la fracción alterada de triglicéridos (Márquez-Ruiz et al., en prensa), la disminución observada en la digestibilidad de los ácidos grasos no alterados podría deberse a que, en parte, se

Tabla IV

Insaponificable: Cantidades excretadas durante 15 dias y digestibilidades corregidas

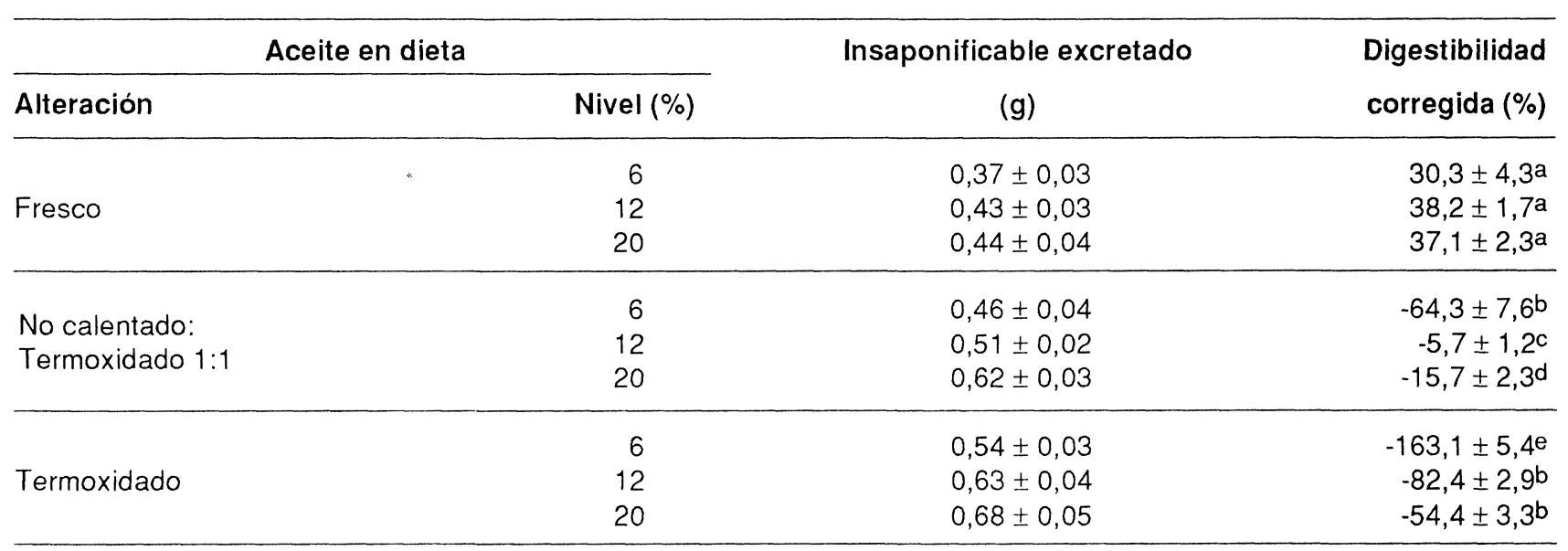

Valores medios \pm error standard de la media.

Los valores de digestibilidad con diferente superindice son significativamente diferentes $(P \leq 0,05)$ 
Tabla V

Acidos grasos no alterados: Cantidades excretadas durante 15 días y digestibilidades corregidas.

\begin{tabular}{|c|c|c|c|}
\hline \multicolumn{2}{|l|}{ Aceite en dieta } & \multirow{2}{*}{$\begin{array}{c}\text { Acidos grasos } \\
\text { excretados } \\
\text { (g) }\end{array}$} & \multirow{2}{*}{$\begin{array}{l}\text { Digestibilidad } \\
\text { corregida (\%) }\end{array}$} \\
\hline Alteración & Nivel (\%) & & \\
\hline \multirow{3}{*}{ No calentado } & 6 & $0,20 \pm 0,07$ & $99,5 \pm 0,1 \mathrm{a}$ \\
\hline & 12 & $0,57 \pm 0,04$ & $99,0 \pm 0,2^{a}$ \\
\hline & 20 & $0,50 \pm 0,04$ & $98,9 \pm 0,2^{a}$ \\
\hline \multirow{3}{*}{$\begin{array}{l}\text { No calentado: } \\
\text { Termoxidado } 1: 1\end{array}$} & 6 & $0,79 \pm 0,11$ & $95,3 \pm 0,7 b$ \\
\hline & 12 & $1,22 \pm 0,13$ & $96,1 \pm 0,7 b$ \\
\hline & 20 & $1,62 \pm 0,13$ & $95,8 \pm 1,0 b$ \\
\hline \multirow{3}{*}{ Termoxidado } & 6 & $1,36 \pm 0,03$ & $89,0 \pm 0,6 c$ \\
\hline & 12 & $2,70 \pm 0,09$ & $88,4 \pm 0,7 \mathrm{c}$ \\
\hline & 20 & $3,83 \pm 0,24$ & $87,8 \pm 1,2^{c}$ \\
\hline
\end{tabular}

Valores medios \pm error standard de la media.

Los valores de digestibilidad con diferente superindice son significativamente diferentes $(P \leq 0,05)$.

encuentran incluidos en moléculas de triglicéridos alterados que podrían ser sólo parcialmente hidrolizados y, en consecuencia, no absorbidos.

En resumen, los resultados obtenidos en este estudio permiten deducir las siguientes conclusiones:

1. La digestibilidad de la grasa total disminuye con el incremento de la alteración, aunque no existen diferencias significativas debidas al nivel de grasa en la dieta.

2. El incremento de alteración de la grasa de la dieta origina una disminución en la absorción de los ácidos no alterados. Por tanto, la menor digestibilidad de la grasa termoxidada no se debe sólo a los compuestos de alteración presentes sino, al mismo tiempo, a un menor aprovechamiento de las moléculas de ácidos grasos no alterados.

3. Los valores negativos de digestibilidad corregida encontrados para la fracción insaponificable en los animales alimentados con aceites alterados demuestran que las cantidades de insaponificable de origen endógeno presentes en los lípidos no absorbidos son superiores a las obtenidas cuando se administra una dieta exenta de grasa. Además, es evidente que la alteración de la grasa contribuye significativamente a aumentar la cantidad excretada de insaponificable de origen endógeno.

\section{AGRADECIMIENTO}

A la Junta de Andalucía y a la Comisión Interministerial de Ciencia y Tecnología (Proyecto ALI 88-0208), por la financiación aportada. A la Sra. Mercedes Giménez, por la ayuda prestada.

\section{BIBLIOGRAFIA}

Boletín Oficial del Estado n.²6, 31 de enero de 1989.

Combe, N., Constantin, M.J. y Entressangles, B. (1981).- "Lymphatic absorption of nonvolatile oxidation products of heated oils in the rat".Lipids 16, 9-14

Dobarganes, M.C., Pérez-Camino, M.C. y Gutièrrez González-Quijano, R. (1984).- "Métodos analíticos de aplicación en grasas calentadas. I. Determinación de ésteres metilicos no alterados".- Grasas y Aceites 35, 172-177.

Deuel, H.J.- "Lipids, vol. II".- Interscience Publisher Inc., New York, 1957.

Firestone, D., Stier, R.F. y Blumenthal, M.M. (1991).- "Regulation of Frying Fats and Oils".- En "Overview. Ouststanding symposie in food science and technology". Institute of Food Technology, Chicago, 90-95.

Hauman, B.F. (1987).- "Trends in frying fat usage".- J. Am. Oil Chemists' Soc. $64,789-805$.

Hoet, P.P., Joosens, J.V., Evrard, E., Eyssen, H. y De Somer, P. (1963)."Intestinal bacteria and faecal fat" en Biochemical Problems of Lipid. Ed. Frazer, A.C.- Elsevier Publishing Company.- Amsterdan, London, New York, 73-83

Hsieh, A. y Perkins, E.G. (1977).- "Nutritional and metabolic studies of methyl esters of dimeric fatty acids in the rat".- Rev. Fr. Corps Gras 24, $19-25$

Iwaoka, W.T. y Perkins, E.G. (1978).- "Metabolism and lipogenic effects of the cyclic monomers of methyl linoleate in the rat".- J. Am. Oil Chemists' Soc. 55, 734-738.

Long, T.T., Jakoi, L., Stevens, R. y Quarfordt, S. (1978).- "The sources of rat biliary cholesterol and bile acids".- Journal of Lipid Research 19, 872-878.

Márquez-Ruiz, G. (1989).- "Evaluación analítica y nutricional de grasas comestibles termoxidadas". Tesis Doctoral. Química Orgánica y Farmacéutica. Facultad de Farmacia. Universidad de Sevilla.

Márquez-Ruiz, G., Pérez-Camino, M.C. y Dobarganes, M.C. (1990).- "Combination of adsorption and size-exclusion chromatography for the determination of fatty acids monomers, dimers and polymers".- Journal of Chromatography 514, 37-44.

Márquez-Ruiz, G., Pérez-Camino, M.C. y Dobarganes, M.C. (1990).- "Evaluación nutricional de grasas termoxidadas y de fritura".- Grasas y Aceites 41, 432-439

Márquez-Ruiz, G., Pérez-Camino, M.C. y Dobarganes, M.C.- "In vitro action of pancreatic lipase on complex glycerides from thermally oxidized oil".- Fat. Sci. Technol. (En prensa).

Márquez-Ruiz, G.. Pérez-Camino, M.C., Ruiz-Gutiérrez, V. y Dobarganes, M.C. (1991).- "Absorción de grasas termoxidadas. I. Reproducibilidad y exactitud de las técnicas analíticas previas a la evaluación de los lipidos no absorbidos".- Grasas y Aceites 42, 32-37.

Metcalfe, L.D. y Schmitz, A.A. (1961).- "The rapid preparation of fatty acid esters for gas chromatographic analysis".- Anl. Chem. 33, 363-364.

Miettinen, T.A., Proia, A. y Mc Namara, D.J. (1981).- "Origins of fecal neutral steroids in rats".- Journal of Lipid Research 22, 485-495.

Orthoefer, F.T. (1987).- "Oil use in the food service industry".- J. Am Oil Chemists' Soc. 64, 795-799.

Perkins, E.G. y Taubold, R. (1978).- "Nutritional and metabolic studies of noncyclic dimeric fatty acid methyl esters in the rat".- J. Am. Oil Chemists' Soc. 55, 632-634.

Potteau, B., Grandgirard, A., Lhuissier, M. y Causeret, J. (1977).- "Recherches récents sur les effects physiopathologiques d'huiles vegétales chauffées".- Bibl. Nutr. Dieta 25, 122-133.

Potteau, B Lhuissier, M. Le Clerc, J Custot, Mezonnet, R y Cluzan, R (1970).- "Recherches sur la composition et les effects physiologiques de l'huile de soja chauffée et de différentes fractions obtenues á partir de cette huile".- Rev. Fr. Corps Gras. 17, 143-153.

Steverink, A.T.G., De Vries, A.W. y Janssen, W.M.M.A. (1985).- "Influence of the composition of fat in poultry diets on the flavour of poultry meat" en "Strategies in food quality assurance: analytical, industrial and legal aspects".- Ed. Baltes W. De Sikkel, Bélgica II, 108-114.

Toullec, R., Flauncy, J. y Rigaud, J. (1968).- "Estimation of lipids in feces. Separate extraction amount and fatty acid composition of unsaponified lipids and insoluble complexes".- Ann. Biol. Anim. Bioch. Biophys. $8,281-289$

(Recibido: Noviembre 1991) 